\title{
EFECTO HIPOGLICEMIANTE DEL EXTRACTO ACUOSO ÁCIDO ATOMIZADO DE Stevia rebaudiana BERTONI EN RATAS ALBINAS
}

\section{Hypoglycemic effect of a tablet produced from the atomized extract of Stevia rebaudiana Bertoni in albino rats}

Mercedes Yance ${ }^{1}$, Marco Aronés ${ }^{1,2}$, Emilio Ramírez ${ }^{1}$, Juan Paniagua ${ }^{1,2}$, Fritz Choquesillo ${ }^{3}$, Américo Castro ${ }^{3}$

${ }^{1}$ Escuela de Formación Profesional de Farmacia y Bioquímica Universidad Nacional de San Cristobal de Huamanga. ${ }^{2}$ Centro de Desarrollo, Análisis y Control de Calidad de Medicamentos y Fitomedicamentos (CEDACMEF), Universidad Nacional de San Cristóbal de Huamanga. ${ }^{3}$ Instituto de Ciencias Farmacéuticas y Recursos Naturales, Universidad Nacional Mayor de San Marcos

\section{RESUMEN}

La Stevia rebaudiana Bertoni es una especie con propiedades edulcorantes, y utilizada como un producto alternativo al azúcar y los endulzantes artificiales. El objetivo de la investigación fue evaluar la acción hipoglicemiante de una tableta elaborada con el extracto atomizado de hojas de Stevia rebaudiana Bertoni. El efecto hipoglicemiante se determinó utilizando el test de tolerancia oral a la glucosa, para el cual se ratas macho, cepa holtzman, con peso promedio de $210 \pm 10 g$, divididas en 5 grupos de a 6 individuos cada uno, aplicándose a cada grupo un tratamiento diferente. Se evaluó la variación de los niveles de glucosa en función del tiempo, se calculó el área bajo la curva (AUC) de los diferentes tratamientos. La tableta elaborada a base del extracto atomizado de Stevia presentó un AUC de 210,68 \pm 57, similar al de insulina que tiene un AUC de $247,43 \pm 24,2$. De las comparaciones múltiples de las medias de las AUC, utilizando la prueba de Duncan al $95 \%$ de nivel de confianza, se determinó que el AUC de los tratamientos con insulina, normoglicemicos, stevia y glibenclamida son estadísticamente similares $(p<0,05)$, aunque existe mayor similitud estadística entre el tratamiento con glibenclamida y el que emplea hiperglicémicos $(p<0,05)$. Se concluyó que el extracto atomizado de hojas de Stevia rebaudiana Bertoni incorporado en tabletas, posee efecto hipoglicemiante.

Palabras clave: tableta, extracto atomizado, Stevia rebaudiana Bertoni, efecto hipoglicemiante.

\section{SUMMARY}

Stevia rebaudiana Bertoni is an specie with sweetening properties, and a product used as alternative to sugar and artificial sweeteners. The objective of the research was to evaluate the hypoglycemic effect of a tablet made with the atomized extract of leaves of Stevia rebaudiana Bertoni. The hypoglycemic effect was determined using the glucose tolerance test, for which it was used o5 male rats, holtzman strain, average weight $210 \pm 10$ g, divided in 5 groups with 6 specimens each, applying to each group a different treatment. The change in glucose levels versus time were evaluated, the area under the curve (AUC) of the different treatments was calculated. The tablet produced from the atomized stevia extract showed an AUC of 210,68 \pm 57, similar to insulin with an AUC of 247,43 $\pm 24,2$. From multiple comparisons of mean AUC, using the Duncan test at 95\% confidence level, it was determined that the AUC(s) of insulin therapy, normoglycemic, stevia and glibenclamide are statistically similar $(p<0,05)$ although treatment with glyburide is statistically similar to hyperglycemic group $(p<0,05)$. It was concluded that the tablet produced from atomized leaves of Stevia rebaudiana Bertoni extract has hypoglycemic effect.

Keywords: Tablet, atomized extract, Stevia rebaudiana Bertoni, hypoglycemic effect.

\section{INTRODUCCIÓN}

a stevia es un género de planta con poder edulcorante, la cual es utilizada como un producto alternativo al azúcar y a los endulzantes artificiales. Es un pequeño arbusto originario de la flora sudamericana que crecía espontáneamente en el hábitat semiárido de las laderas montañosas de Paraguay y en las zonas adyacentes de Brasil. Esta planta no tiene calorías y posee efectos beneficiosos en la absorción de la grasa y la presión arterial. Contiene carbohidratos, proteínas, vitaminas y minerales, por lo que hoy en día, en países como Japón abarca el $41 \%$ de los edulcorantes consumidos. El sabor dulce de la planta se debe a un glucósido llamado esteviósido, además de otros compuestos como glucosa y rebaudiósido ${ }^{(1)}$.

La stevia en su forma natural es 15 veces más dulce que el azúcar de mesa (sucrosa) y el extracto es de 100 a 300 veces más dulce aún. Algo importante de recalcar es que se ha descubierto que no afecta los niveles de azúcar sanguíneo, por el contrario, posee propiedades hipoglucémicas, las cuales mejoran la tolerancia a la glucosa. Por este motivo se recomienda su uso a pacientes diabéticos ${ }^{(2)}$.

La diabetes mellitus es un problema de salud pública importantequeafecta a gran parte de la población mundial y nacional. En las Américas se las cifras y proyecciones respecto al número de personas con diabetes mellitus son de 35 millones en el año 200o, de 40 millones el 2005 y se espera que alcancen a los 63 millones para el año $2025^{(3)}$.

En ese sentido, se planteó el objetivo de elaborar una tableta a base del extracto atomizado de hojas de Stevia rebaudiana Bertoni, para evaluar su efecto hipoglicemiante en ratas albinas, utilizando el método del test de tolerancia oral a la glucosa ${ }^{(4,5)}$.

\section{MATERIAL Y MÉTODOS}

Material biológico. Las hojas de Stevia rebaudiana Bertoni, fueron recolectadas del bosque húmedo tropical de la comunidad de Palestina Baja, del distrito de Kimbiri, Provincia de La Convención, Departamento de Cusco.

Se emplearon 30 ratas albinas, de $200 \pm 10 \mathrm{~g}$ de peso, adquiridas del Bioterio de la Universidad Nacional 


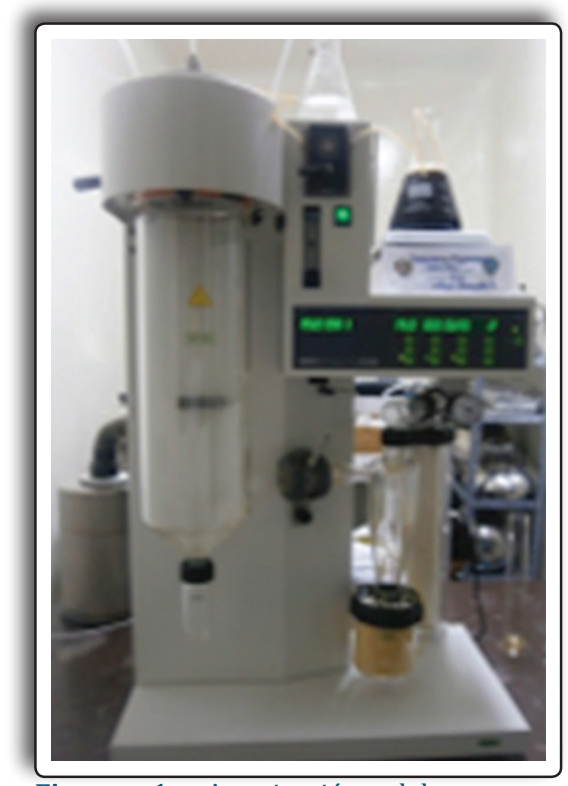

Figura 1. Atomización del extracto atomizado de Stevia rebaudiana.

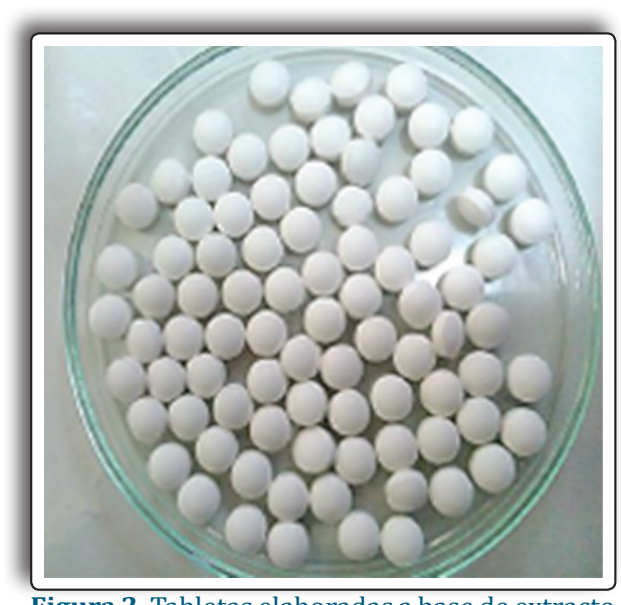

Figura 2. Tabletas elaboradas a base de extracto atomizado de Stevia rebaudiana Bertoni.

Agraria La Molina, alojados y aclimatados en jaulas individuales a temperatura ambiental entre 23 a $25^{\circ} \mathrm{C}$ y una humedad relativa menor a $70 \%$.

Obtención del extracto atomizado. Un kilo de hojas fueron desecadas y reducidas en molino de cuchillas con malla de $1 \mathrm{~cm}$ aproximadamente; luego se sometió a lixiviación utilizando 20 litros de solución de ácido cítrico al 10\% en agua destilada a temperatura ambiente. La solución enfriada a $2{ }^{\circ} \mathrm{C}$ se filtró por membranas de 0,1-0,2 mm. El extracto se concentró por evaporación, en rotavapor industrial, a menos de $45^{\circ} \mathrm{C}$, hasta obtener un 10\% de sólidos totales.

La solución concentrada, mezclada con maltodextrina como vehículo, se secó utilizando atomizador Spray Driver B290 (figura 1) a temperatura de entrada 170 a $180^{\circ} \mathrm{C}$, temperatura de salida 77 a $83^{\circ} \mathrm{C}$, aspirador de $100 \%$, porcentaje de bomba de 15 a $30 \%$ y flujo de muestra de $3-4 \mathrm{~cm}^{3} / \mathrm{min}$.

Para la obtención del extracto atomizado se siguió el procedimiento descrito por Razo ${ }^{(6)}$.

\section{Evaluación de los parámetros fisicoquímicos del extracto atomizado}

Se evaluaron las características organolépticas, identificación fitoquímica (Miranda M. y Cuéllar A.) (7), solubilidad, $\mathrm{pH}$, humedad y cenizas totales.

\section{Formulación de las tabletas}

Método de elaboración de las tabletas. Se cernió celulosa microcristalina (PH-101), carboximetilcelulosa sódica, dióxido de silicio y extracto atomizado, por un tamiz de o,4 $\mathrm{mm}$ de luz. Se mezcló en mezclados en "V" por 10 minutos, se adicionó el lubricante y se tamizó pasó por tamiz de diámetro o,4 mm. Se llevó nuevamente a mezclador en "V" por 10 minutos. Se realizó la compresión en tableteadora monopunzón, con un parámetro de 60 golpes por minuto ${ }^{(8)}$.

Controles. Se midió el tamaño, y la uniformidad de peso -se pesaron 10 tabletas haciendo uso de la balanza analítica y desintegración-.
Valoración de los niveles de esteviósido en la tableta. Se tomaron en cuenta las directrices de la USP (9) empleando la metodología analítica sugerida por Joint FAO/WHO Expert Committee on Food Additives (10). Se utilizó equipo de Cromatografía Líquida de Alta Resolución (HPLC) marca DIONEX ULTIMATE 3000. La solución estándar se preparó utilizando $50 \mathrm{mg}$ de estándar disolviendo y enrasando con agua-acetonitrilo (7:3) a $50 \mathrm{~mL}$. La solución de muestra se preparó utilizando 50-100 mg de muestra disolviendo y enrasando con aguaacetonitrilo (7:3) a $50 \mathrm{~mL}$. Los parámetros fueron: longitud de onda de 210 j; volumen de inyección de $5 \mu$ l de solución estándar y de muestra; columna C18 (longitud: $250 \mathrm{~mm}$; diámetro interior: 4,6 mm y tamaño de partícula $5 \mu \mathrm{m}$ ); temperatura $40^{\circ} \mathrm{C}$; fase móvil de acetonitrilo y buffer de fosfato de sodio $10 \mathrm{mmol} / \mathrm{L}(\mathrm{pH} 2,8)$ en proporción 32:68 y flujo de $1 \mathrm{~mL} / \mathrm{min}$.

El porcentaje del esteviósido en la muestra se calculó a partir de la fórmula:

$$
\% X=\left(\frac{W_{\mathrm{s}}}{W}\right) \cdot\left(\frac{f_{\mathrm{x}} \cdot A_{\mathrm{x}}}{A_{\mathrm{s}}}\right) \cdot 100
$$

Donde:

$\% \mathrm{x}=$ Porcentaje del esteviósido

$A=$ Área del pico

$W=$ Cantidad $(\mathrm{mg})$

$\mathrm{s}=$ Esteviosido

$X=$ Solución muestra

$f_{\mathrm{x}}=$ Relación del peso de la fórmula de $X$ para el peso de la fórmula de esteviósido: 1.00 (esteviósido).

\section{Evaluación de la actividad hipoglucemiante}

Se utilizó el test de tolerancia oral a la glucosa, según CYTED 1995 y Arroyo $2012^{(4,5)}$.

El nivel de glucosa se midió utilizando el glucómetro digital, para lo cual se tomó la muestra de sangre de la cola usando una lanceta.

Las tabletas, la insulina y la glibenclamida, por separado, se disolvieron en agua destilada y se administraron inmediatamente después de la glucosa por vía oral, a excepción de la insulina que se administró por vía subcutánea.

Paralelamente al tratamiento se administró glucosa por vía intraperitoneal en dosis de $200 \mathrm{mg} / \mathrm{kg}$. Las mediciones de glucosa se hicieron antes de iniciar los tratamientos y a diferentes tiempos después de la administración de glucosa.

Se graficaron los niveles de glucosa a diferentes tiempos y se calculó el área bajo la curva de niveles plasmáticos en función del tiempo, utilizando el método de los trapecios.

$$
A=\frac{1}{2}\left(f_{(a)}+f_{(b)}\right) \cdot(b-a)
$$


Tabla 1. Aleatorización de las ratas en distintos grupos de tratamiento.

\begin{tabular}{ccc}
\hline Grupos & Tratamiento & Dosis \\
\hline 1 & Suero fisiológico & $2 \mathrm{ml} / \mathrm{Kg}$ \\
2 & Glucosa & $2000 \mathrm{mg} / \mathrm{Kg}$ \\
3 & Glucosa + Glibenclamida (Glib.) & $50 \mathrm{mg} / \mathrm{kg}$ \\
4 & Glucosa + Insulina & $4 \mathrm{UI} / \mathrm{Kg}$ \\
5 & Glucosa +Tableta de stevia & $100 \mathrm{mg} / \mathrm{Kg}$ \\
\hline
\end{tabular}

\section{Diseño experimental}

Alcance de investigación. Experimental ${ }^{(11)}$.

Diseño de investigación. Diseño con pos prueba únicamente y grupo control

$\mathrm{R}$ : Asignación al azar o aleatoria

$$
\begin{aligned}
& R G_{1} \times O_{1} \\
& R G_{2}-O_{2}
\end{aligned}
$$

$\mathrm{G}$ : Grupos de ratas.

$\mathrm{X}$ : Tratamiento, estímulo o condición experimental.

O: Medición experimental: Área bajo la curva.

\section{Análisis de datos}

La comparación de los promedios del AUC de los diferentes tratamientos fueron comparados a través del análisis de varianza (ANOVA) al 95\% de nivel de confianza y la prueba de comparaciones múltiples de Duncan.

\section{RESULTADOS}

Los resultados se muestran en las tablas 2-6 y en las figuras 3-5.

\section{DISCUSIÓN}

La tabla 2 muestra un porcentaje de rendimiento de $12,5 \%$, es decir de $1 \mathrm{~kg}$ de corteza se obtiene $125,6 \mathrm{~g}$ de extracto atomizado, resultado menor al obtenido por Razo (6). Esta variación podría ser debida al lugar de procedencia de la planta, ya que esta suele cultivarse a temperaturas entre los 23 y $25^{\circ} \mathrm{C}$ con humedad relativa menor a $70 \%$, crece entre los 500 y 1600 de altitud y su hábitat es en zonas con mucha luz solar, pero poco caliente.

Respecto a las características, el extracto presenta olor dulce, sabor dulce y aspecto polvo fino homogéneo, específicos para los extractos atomizados de stevia, mientras que el color beige difiere de los obtenidos por Razo ${ }^{(6)}$, debido al proceso de ultrafiltración que él utilizó. El extracto atomizado es muy soluble en agua, poco soluble en metanol y prácticamente insoluble en cloroformo. El extracto tiene un $\mathrm{pH}$ igual a 5,70, ligeramente ácido, y humedad de $3,57 \%$, menor a la obtenida por Razo ${ }^{(6)}$, pero dentro de las especificaciones, pues un exceso de humedad puede provocar crecimiento microbiano o hidrólisis de algunos metabolitos, como manifiestan Miranda ${ }^{(7)}$ y Sharapin ${ }^{(8)}$. Presenta cenizas totales igual a 4,66\%, mayor a la obtenida por Razo ${ }^{(6)}$, posiblemente debido a materias extrañas -principalmente suelo y arena- que se adhieren a superficie de las hojas.

Los metabolitos secundarios presentes en el extracto atomizado fueron flavonoides, fenoles, taninos, triterpenos y esteroides (tabla 3), lo que corrobora los hallazgos de Bravo ${ }^{(12)}$. Esto demuestra que en el proceso de secado por atomización no se pierden dichos
Tabla 2. Parámetros fisicoquímicos del extracto atomizado de las hojas de Stevia rebaudiana Bertoni.

\begin{tabular}{lcc}
\hline \multicolumn{1}{c}{ Parámetros } & Ensayos & Resultados \\
\hline \multirow{3}{*}{ Organoléptico } & Color & Beige \\
& Olor & Dulce \\
& Sabor & Dulce \\
& Aspecto & Polvo fino homogéneo \\
& Agua & Muy soluble \\
Solubilidad & Metanol & Poco soluble \\
& Cloroformo & Insoluble \\
pH & E.A. al 5\% & 5,7 \\
Humedad & Gravimétrico & $3,57 \%$ \\
Cenizas & Gravimétrico & $4,66 \%$ \\
Rendimiento & & $12,50 \%$ \\
\hline
\end{tabular}

E.A.: Extracto atomizado

Tabla 3. Parámetros fisicoquímicos del extracto atomizado de las hojas de Stevia rebaudiana Bertoni.

\begin{tabular}{lccc}
\hline \multicolumn{1}{c}{ Metabolito } & Ensayos & Resultados & Obs. \\
\hline Flavonoides & Shinoda & + & Fase amílica anaranjado \\
$\begin{array}{l}\text { Fenoles y/o } \\
\text { taninos }\end{array}$ & $\begin{array}{c}\text { Cloruro } \\
\text { férrico }\end{array}$ & +++ & Azul verdoso \\
Triterpenos & $\begin{array}{c}\text { Libermann } \\
\text { - Burchad }\end{array}$ & +++ & Verde oscuro \\
\hline
\end{tabular}

(+) Escasa; (++) Buena; (+++) Excelente

Tabla 4. Formulación de una tableta elaborada a base de extracto atomizado de Stevia rebaudiana Bertoni.

\begin{tabular}{lc}
\multicolumn{1}{c}{ Principios y excipientes } & $\%$ \\
\hline E.A. de Stevia rebaudiana Bertoni & 48,89 \\
Celulosa microcristalina (PH-101) & 48,22 \\
Carboximetil celulosa sódica & 1,89 \\
Dióxido de silicio & 0,76 \\
Esterato de magnesio & 0,24 \\
\hline
\end{tabular}

E.A.: Extracto atomizado

Tabla 5. Controles realizados a la tableta elaborada con del extracto atomizado de Stevia rebaudiana Bertoni.

\begin{tabular}{lc}
\hline \multicolumn{1}{c}{ Controles } & Parámetro \\
\hline Diámetro (mm) & 8,05 \\
Altura (mm) & 5,46 \\
Uniformidad de peso (mg) & 205,02 \\
Desintegración (min) & 14,97 \\
\hline
\end{tabular}

Tabla 3. Valoración de los niveles de esteviósido presentes en la tableta de Stevia rebaudiana Bertoni.

\begin{tabular}{lcccc}
\hline & TR & Área & ES & C/tab \\
\hline Estándar & 8,87 & 16,06 & & \\
Muestra & 9,04 & 0,329 & 2,33 & 4,77 \\
\hline
\end{tabular}

TR: Tiempo de retención ( $\mathrm{min})$

ES: esteviósidos (\%) C: concentración (mg) Tab: Tableta

metabolitos, responsables del efecto farmacológico, específicamente los diterpenos, que muchos estudios reportan como responsables del efecto hipoglicemiante.

Trillo ${ }^{(13)}$, manifiesta que un estudio de formulación debe ir precedido del conocimiento de determinadas propiedades físicas, químicas y biofarmacéuticas del principio activo y de la influencia de los excipientes y el proceso tecnológico sobre estos, para conseguir las tres cualidades fundamentales del medicamento que son la estabilidad, seguridad y eficacia. Asimismo, González et al ${ }^{(14)}$, expresan que la elaboración de un comprimido por el método de la compresión directa presenta algunos inconvenientes potenciales, como la tendencia a la dureza 


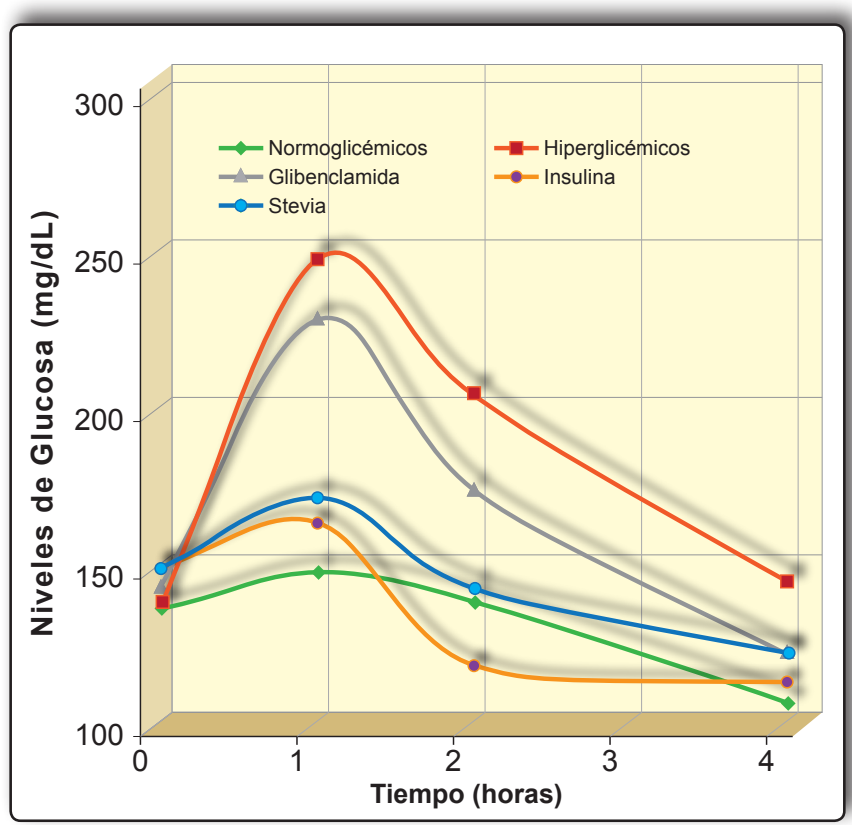

Figura 3. Variación de los niveles medios de glucosa de los tratamientos en función del tiempo.

elevada y a la adherencia, el tiempo de disgregación excesivo y problemas asociados al flujo irregular. En el presente estudio esta situación podría agravarse debido a la higroscopicidad inherente a los extractos atomizados y a la cantidad de extracto añadida. La medida utilizada para contrarrestar dichos problemas en la elaboración de tabletas a base del extracto atomizado Stevia rebaudiana Bertoni fue el empleo de coadyuvantes derivados de la celulosa en el proceso por compresión directa.

Para la elaboración de las tabletas se tomó como referencia la fórmula propuesta por González et al (14), cuya composición se presenta en la tabla 4 .

Los controles realizados a las tabletas se presentan en la tabla 5, observándose que cumplen con los estándares permitidos, al presentar uniformidad en el diámetro y altura, ya que el rango de variación permitido es de $\pm 5 \%$; asimismo, estas dimensiones físicas del material junto con las densidades de los excipientes determinarán el peso de la tableta, cuyo promedio fue de $205,02 \pm 1,22 \mathrm{mg}$, cumpliendo con los estándares permitidos en lo referente a uniformidad de peso (8). La desintegración sirve como guía en la preparación de una fórmula óptima y en las pruebas de control de proceso para asegurar la uniformidad de lote a lote, el tiempo que tardó en desintegrarse fue de 14,97 minutos, que al igual que los controles anteriores cumplen con los estándares permitidos ${ }^{(15)}$.

Para la valoración de los niveles de esteviósido en la tableta, se tomó como referencia la técnica establecida en la $73^{\text {a }}$ reunión del Comité de Expertos de la FAO/OMS en Aditivos Alimentarios (9). El contenido de esteviósido en cada una de ellas fue de $2,33 \%$, con una concentración de $4,77 \mathrm{mg}$ por tableta, tal como se puede observar en la tabla 6. Los niveles de esteviósido son muy bajos en comparación a los mencionados por Peña ${ }^{(3)}$ y Jiménez et al (16), quienes manifiestan que existe una gran variabilidad para los contenidos de esteviósido y rebaudiósido A, observándose

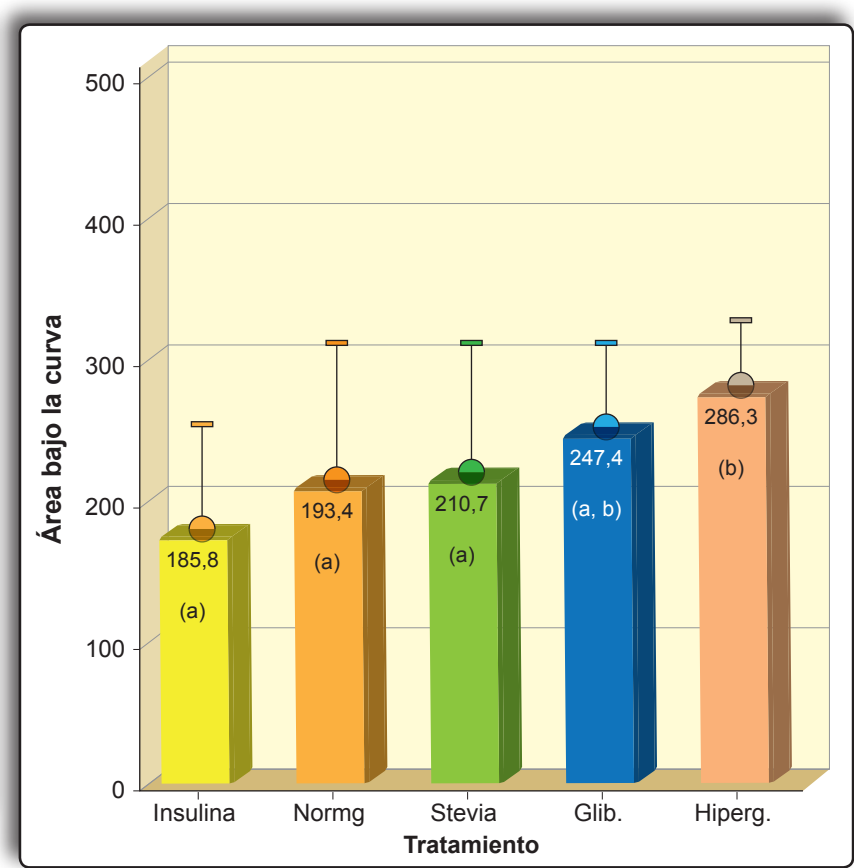

Figura 4. Área bajo la curva de niveles plasmáticos de glucosa de los distintos tratamientos.

valores de $0-21 \%$ para el primero, y o a $12 \%$ para el segundo. Dicha variabilidad se debe, como menciona Jiménez et al (16), a que estevia se reproduce sexualmente por fecundación cruzada (alógama), por lo que no debe extrañar la diversidad fenotípica y genotípica que se observa tanto en plantas que crecen espontáneamente, como en cultivos comerciales; ambas características son gobernadas por genes de herencia cuantitativa, por lo que es de esperar influencias de factores ambientales sobre los mismos (época de cosecha, pos cosecha, temperatura, humedad, etc.)

Respecto a la tolerancia oral a la glucosa, la figura 3 muestra los niveles de glicemia en función del tiempo para los distintos tratamientos, donde podemos observar que la hiperglicemia ( $\geq 200 \mathrm{mg} / \mathrm{dL}$ ) perdura por más de dos horas después de la ingesta de $2000 \mathrm{mg} / \mathrm{Kg}$ de glucosa por vía oral (grupo 2, hiperglicémicos). Asimismo se observa que la tableta elaborada a base del extracto atomizado de Stevia rebaudiana Bertoni, reduce los niveles de glicemia a las dos horas $(142 \pm 39 \mathrm{mg} / \mathrm{dL})$ en comparación al control glibenclamida $(173 \pm 37 \mathrm{mg} / \mathrm{dL})$.

Jeppesen et al ${ }^{(16)}$ y Gregersen et al ${ }^{(17)}$ determinaron el efecto antihiperglucémico de la Stevia rebaudiana Bertoni, utilizando el área bajo la curva (AUC) de los niveles de glicemia. Para determinar el área bajo la curva se utilizó el método de los trapecios, observando que la tableta elaborada a base del extracto atomizado de Stevia rebaudiana Bertoni presenta un AUC de 210,68 \pm 57, menor al AUC del control glibenclamida que obtuvo un área de 247,43 \pm 33 .

Al realizar el análisis devarianza de las medias del AUC de los niveles glicemia en función del tiempo de los tratamientos, se determinó que existen diferencias estadísticamente significativas $(p<0,05)$ entre diversos tratamientos. Seguidamente se realizaron comparaciones múltiples de las medias de las AUC utilizando la prueba de Duncan al 95\% de 
nivel de confianza, los resultados se muestran en la tabla 7 , donde se puede observar que los AUC de los tratamientos con insulina, normoglicemicos, Stevia rebaudiana y glibenclamida son estadísticamente similares ( $p<0,05)$, aunque el tratamiento con glibenclamida es estadísticamente similar en el grupo de los hiperglicémicos $(p<0,05)$.

Todos estos resultados nos conducen a afirmar que la tableta elaborada a base del extracto atomizado de hojas de Stevia rebaudiana Bertoni, podría ejercer actividad hipoglicemiante estadísticamente significativa con el grupo tratado con insulina y glibenclamida. Esta afirmación es sustentada por la buenas características fisicoquímicas de la tableta, su menor tiempo de desintegración y la presencia de esteviósido y rebaudiósido A en ella.

Muchos estudios reportaron disminución estadísticamente significativa de la acción hipoglicemiante, atribuyendo este efecto principalmente al esteviósido, metabolito presente en la Stevia rebaudiana Bertoni como refieren Jeppesen et al ${ }^{(17)}$ y Gregersen et al ${ }^{(18)}$. El presente estudio refuerza tal hipótesis pues se ha demostrado que el extractoatomizadode Stevia rebaudiana Bertoniquecontiene esteviósido y podría tener actividad hipoglicemiante.

Jeppesen et al (17), investigaron profundamente al esteviósidocomometabolitoactivoenelefectohipoglicemiante, para lo cual utilizaron líneas celulares beta INS-1 de islotes de Langerhans de ratón, describiendo el posible mecanismo de acción, a través de la estimulación de la secreción de insulina por acción directa en las células beta pancreáticas.

\section{CONCLUSIÓN}

La tableta elaborada a base de extracto atomizado de hojas de Stevia rebaudiana Bertoni, posee acción hipoglicemiante en ratas albinas.

\section{REFERENCIAS BIBLIOGRÁFICAS}

1. Rodríguez J, Sáenz M. Obtención de un edulcorante natural proveniente de la stevia (Stevia rebaudiana Bertoni). [Internet]. Costa Rica: Universidad Earth; 2005 [Citado 12 Setiembre 2013]. Disponible en: http://usi.earth.ac.cr/glas/sp/dpg/200533.pdf

2. Stevia Canadá. Learning From Ancient Wisdom. [Internet]. Canadá; 2005 [Citado 12 Setiembre 2013]. Disponible en: http://www.steviacanada.com/

3. Peña N. La Stevia rebaudiana y su propiedad hipoglicemiante en pacientes con diabetes mellitus tipo 2 realizado en el Instituto de Gastroenterologia Boliviano Japonés. Universidad Mayor de San Andrés. [Internet]. Bolivia; 2006 [Citado 12 de Setiembre del 2013]. Disponible en: http://bibliotecadigital.umsa.bo:808o/ rddu/bitstream/123456789/520/1/TN954.pdf

4. Arroyo J, Cisneros C. Modelos Experimentales de Investigación Farmacológica. Lima: ASDIMOR S.A.C.; 2012.

5. CYTED. Manual de Técnicas de Investigación. Programa Iberoamericano de Ciencia y Tecnología para el Desarrollo. Proyecto X-1; Búsqueda de Principios Activos en Plantas de la Región; 1995.

6. Razo E. Diseño de una planta piloto para la industrialización de stevia en la Comunidad Cueva de los Monos, Cantón Sacha, Provincia de Orellana. [Tesis].
Facultad de Ingeniería Química y Agroindustria de la Escuela Politécnica Nacional. Quito; 2011.

7. Miranda M. Métodos de Análisis de Drogas y Extractos. La Habana: Instituto de Farmacia y Alimentos-Universidad de la Habana. 2002.

8. Sharapin N. Fundamentos de Tecnología de Productos Fitoterapéuticos. Convenio Andrés Bello (CAB) y el Programa Iberoamericano de Ciencia y Tecnología; 2000.

9. The Official Compendia of Standards USP 35 NF 30. The United States Pharmacopoeia. -The National Formulary. Maryland: Pharmacopeial Convention Inc.; 2012.

10. Joint FAO/WHO Expert Committee on Food Additives. Compendium of Food Additive Specifications. [Internet]. Roma: FAO; 2010 [Citado o1 de Abril del 2014]; Disponible en: http://www.fao.org/docrep/o13/i1782e/i1782e.pdf

11. Hernández R, Fernández C, Baptista L. Metodología de la Investigación Científica. $4^{\mathrm{a}}$ ed. México: McGraw-Hill Interamericana; 2006

12. Bravo A, Ale B, Rivera C, Huamán M, Delmás R, Rodríguez B et al. Caracterización Química de la Stevia Rebaudiana. Rev Per Quím Ing [Serie en internet]. 2009 [Citado 12 Setiembre 2013]; 12(2): 5-8. Disponible en: http://sisbib.unmsm.edu.pe/ bibvirtual/publicaciones/ing_quimica/v12_n2/pdf/aoıv12.pdf

13. Trillo F. Tratado de Farmacia Galénica. Madrid: Luzán S.A.; 1993.

14. González G,SchmidtP.Obtencióndecomprimidosconteniendo extractos atomizados de Flor de la Pasión (Passiflora incarnata L.). Acta Farm Bonaerense [Serie en internet]. 1995 [Citado o1 Abril 2014]; 14 (3): 173-80. Disponible en: http://www. latamjpharm.org/resumenes/14/3/LAJOP_14_3_1_3.pdf.

15. Gennaro A. Remington Farmacia Tomo 2. $20^{\mathrm{a}}$ ed. Buenos Aires: Editorial Médica Panamericana S.A.; 2003.

16. Jiménez T, Cabrera G, Álvarez E, Gómez F. Evaluación del contenido de esteviósido y rebaudiósido A en una población de Stevia rebaudiana Bertoni (kaâ heê) cultivada comercialmente. Mem Inst Investig Cienc Salud [Serie en internet]. 2010 [Citado 12 Setiembre 2013]; 6 (1): 47-53. Disponible en: http://scielo.iics.una.py/ scielo. php?pid=S1812-95282010000100007 \&script=sci_arttext.

17. Jeppesen P, Gregersen S, Rolfsen S, Jepsen M, Colombo M, Agger A et al. Antihyperglycemic and blood pressurereducing effects of stevioside in the diabetic Goto-Kakizaki rat. Metabolism [Serie de internet]. 2003 [Citado 12 Setiembre 2013]; 52 (3): 372-378. Disponible en: http://www.steviainfo. com/research_articles/Jeppesen\%2oet $\% 20 a l \% 20(2003)$.pdf.

18. Gregersen S, Jeppesen P, Holst J, Hermansen K. Antihyperglycemic effects of stevioside in type 2 diabetic subjects. Metabolism [serie de internet]. 2004 [Citado 12 Setiembre 2013]; 53(1): 73-76. Disponible en: http://www.sciencedirect.co m/science/article/pii/ Soo2604950300387.

Manuscrito recibido el: 14/o8/2015

Aceptado para su publicación el: 02/11/2015

Correspondencia:

Nombre: $\quad$ Fritz Fedor Choquesillo Peña

Dirección: Jr. Puno 1002 Jardín Botánico - Lima

e-mail: $\quad$ fritzchp@hotmail.com

fchoquesillop@unmsm.edu.pe 